\title{
Recurrence of Critical Limb Ischemia After Endovascular Intervention in Patients with Diabetic Foot Ulcers
}

\author{
Marco Meloni, ${ }^{1, *}$ Valentina Izzo, Laura Giurato, \\ Costantino Del Giudice, ${ }^{2}$ Valerio Da Ros, ${ }^{2}$ Valerio Cervelli, \\ Roberto Gandini, ${ }^{1}$ and Luigi Uccioli ${ }^{1}$ \\ Departments of ${ }^{1}$ Systems Medicine, ${ }^{2}$ Interventional Radiology, and ${ }^{3}$ Plastic and Reconstructive Surgery, \\ University of Tor Vergata, Rome, Italy.
}

Objective: To establish the rate of clinical recurrence of critical limb ischemia (CLI) in diabetic patients with ischemic foot ulcers (DFUs) treated by percutaneous transluminal angioplasty (PTA).

Approach: The study group was composed of 304 patients with ischemic DFUs treated by PTA. We evaluated the rate of clinical recurrence of CLI requiring a second PTA (repeated PTA [rePTA]), the factors related to CLI relapse, and the outcomes of rePTA patients. The follow-up was $12.5 \pm 6.6$ months.

Results: Seventy-four of 304 patients $(24.3 \%)$ needed rePTA. The mean time to rePTA was $3.5 \pm 0.64$ months. rePTA group in comparison with no rePTA group had lower rate of healing $(28.5 \%$ vs. $71.9 \% p=0.0001)$, higher rate of ulcer recurrence $(20 \%$ vs. $10.3 \% p=0.03)$, major amputation $(24.3 \%$ vs. $4.3 \%$ $p=0.0005)$, and death $(33.3 \%$ vs. $7.9 \% p=0.002)$. Glycated hemoglobin, type A1C (HbA1c; $2.2[1.9-2.7] p=0.02)$ and dialysis $(1.5[1.4-3.6] p=0.006)$ were independently associated to clinical recurrence of CLI after PTA.

Innovation: To identify the outcomes of patients with clinical recurrence of CLI and the clinical factors involved to reduce the rate of restenosis after endovascular treatment and improve the rate of limb salvage.

Conclusions: Clinical recurrence of CLI is associated with a high rate of nonhealing ulcer recurrence, major amputation, and death. Dialysis and impaired glycemic control were independent predictors of CLI relapse after endovascular treatment.

Keywords: diabetes, diabetic foot ulcers, critical limb ischemia, revascularization, limb salvage

\section{INTRODUCTION}

Peripheral ARTERIAL diseASE (PAD) is a severe complication of diabetes and can be found approximately in $50 \%$ of subjects with diabetic foot ulcers (DFUs). ${ }^{1-3}$ PAD is a strong risk factor for major amputation, ${ }^{4,5}$ and peripheral revascularization, surgical, or endovascular is the only therapeutic option. The role of percutaneous transluminal angioplasty (PTA) in di- abetic patients with critical PAD, especially infrapopliteal disease, is well recognized. ${ }^{6-14}$ Several studies have documented good results in terms of feasibility, technical efficacy, reduced number of complications, and limb salvage rate. Furthermore, PTA can also be performed in patients who cannot be candidates for open surgery due to the presence of several comorbidities. $^{7,8,15,16}$

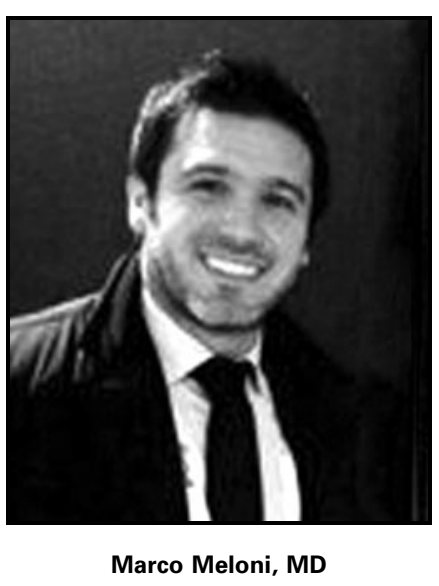

Submitted for publication December 14, 2017. Accepted in revised form December 22, 2017

*Correspondence: Department of Systems Medicine, University of Tor Vergata, Viale Oxford 81, Rome 00133, Italy

(e-mail: meloni.marco@libero.it). 


\section{CLINICAL PROBLEM ADDRESSED}

Despite the great results obtained through endovascular revascularization in the last years, the current problem is the frequent restenosis after PTA, requiring a second approach. In fact, in comparison to by-pass, PTA is characterized by higher rate of restenosis. ${ }^{17-20}$ Although angioplasty can be easily repeated in case of restenosis or reocclusion, ${ }^{21-23}$ the early and frequent recurrence of critical limb ischemia (CLI) influences the clinicians approach and affects the outcomes of patients with ischemic DFUs. In our limb salvage protocol, all patients affected by DFUs and CLI are treated by revascularization, usually by endovascular approach. After hospitalization, they undergo a close follow-up until the ulcer has healed. In case of recurrence of CLI in the affected limb, the patients are considered for a new revascularization repeated PTA (rePTA). The aim of our study was to establish the rate of rePTA in diabetic patients with DFUs and CLI. Then, we evaluated the outcomes of rePTA patients and the specific factors related to the relapse of CLI in these subjects.

\section{MATERIALS AND METHODS}

The study group composed of 304 subjects with DFUs and CLI, who were followed up in our diabetic foot unit. All patients underwent a limb salvage protocol: surgical debridement of infected tissues or gangrene, antibiotic therapy in case of infection, offloading of affected foot, and revascularization. All peripheral revascularizations have been performed through endovascular approach. ${ }^{24}$

An informed verbal consent was obtained from subjects involved in this study.

\section{PTA procedure}

Percutaneous angioplasty was performed in the presence of significant arterial stenosis $(>50 \%$ of lumen) and/or obstruction. In selected patients, self-expandable stents were inserted in 5- to 8-mm diameter vessels. Stents were not placed below the popliteal arteries as the risk of thrombosis was very high in such low-flow vessels. In selected patients, the subintimal approach was used to create a new lumen between intimal and medial layers.

Before and after PTA, the patients were treated by dual antiplatelet therapy (Cardioaspirin $100 \mathrm{mg}$ plus Clopidogrel $75 \mathrm{mg}$ or Ticlopidine $250 \mathrm{mg} \times 2$ in case of intolerance to Cardioaspirin or Clopidogrel) at least for 1 month and after only by Cardioaspirin or Clopidogrel long life. All cardiovascular risk factors (smoke, hypertension, dyslipidemia, and hyperglycemia) were checked and treated if required. According to our protocol, after hospitalization, the patients have been regularly followed up in our Diabetic Foot Unit until ulcer healing. Recurrence of CLI was considered in case of nonhealing (absence of granulation tissue, absence of epithelialization signs, and reduction of ulcer size less than $50 \%$ after at least 4 weeks of standard of care), recurrence of pain, and ulcer relapse in association with transcutaneous oxygen pressure $\left(\mathrm{TcPO}_{2}\right)<30 \mathrm{mmHg}$ and/or positive duplex scanning. If clinical signs of ischemia were supported by instrumental evaluation, the patients were considered for another angiographic evaluation and treated by a new PTA (rePTA) if required.

We evaluated the rate of patients who needed rePTA, the mean time to rePTA, the predictive factors related to the recurrence of CLI and the outcomes according to rePTA or not expressed as healing, major amputation, and death. Healing was considered in case of complete epithelialization of previous ulcers. Amputation above the ankle was considered major amputation. Amputation freesurvival after PTA for rePTA and no rePTA groups was reported by Kaplan-Meier analysis.

\section{Statistical analysis}

Statistical analysis was performed by SAS (JMP12; SAS Institute, Cary, NC) for personal computer. Data are expressed as means \pm SEM. Comparison between groups was reported by a $\chi^{2}$ test (frequency data) or ANOVA (continuous data). Univariate logistic analysis was performed for all potential predictor variables according to the detected outcome. All predictors identified by univariate analysis were evaluated simultaneously in a multivariable regression. $p<0.5$ was considered as statistically significant.

\section{RESULTS}

Table 1 reports the baseline characteristics of the whole population, rePTA group, and no rePTA groups. Three hundred four patients were treated by PTA. In $57(18.7 \%)$ cases a stent was applied in iliac-femoral district; $52(17.1 \%)$ patients were treated by subintimal approach. Fourteen (4.6\%) patients had minor PTA technical complications. In $4 / 304(1.3 \%)$ cases, there was a distal embolization; in 4/304 (1.3\%), an arterial dissection; and in 6/304 (2\%), a retroperitoneal bleeding (hematoma). Three of four patients with distal embolization needed major amputation due to severity of foot ischemia and untreatable pain. The follow-up was $12.5 \pm 6.6$ months.

\section{Restenosis after PTA}

Seventy-four of 304 patients $(24.3 \%)$ needed rePTA after the first treatment. The mean time to rePTA was $3.5 \pm 0.64$ months. In 70/74 (94.2\%) 
Table 1. Baseline characteristics of the whole population, rePTA group, and no rePTA group

\begin{tabular}{|c|c|c|c|c|}
\hline & General & rePTA & No rePTA & $p$ \\
\hline Sex (male) & $72 \%$ & $68.5 \%$ & $73.5 \%$ & 0.64 \\
\hline Age (years) & $68.3 \pm 10.9$ & $67.1 \pm 10.1$ & $68.7 \pm 11.5$ & 0.49 \\
\hline Diabetes (type 2) & $91.1 \%$ & $88.2 \%$ & $91.7 \%$ & 0.55 \\
\hline Diabetes duration (years) & $21 \pm 1.6$ & $20.3 \pm 2$ & $21.4 \pm 1.2$ & 0.63 \\
\hline $\mathrm{HbA1c}(\mathrm{mmol} / \mathrm{mol})$ & $61.4 \pm 22.8$ & $67.6 \pm 23.2$ & $58.6 \pm 21.6$ & 0.05 \\
\hline Smoke & $9 \%$ & $9.3 \%$ & $10 \%$ & 0.91 \\
\hline Hypertension & $79.5 \%$ & $75.8 \%$ & $80 \%$ & 0.61 \\
\hline Dyslipidemia & $64 \%$ & $71.7 \%$ & $62.5 \%$ & 0.23 \\
\hline Dialysis & $31.4 \%$ & $54 \%$ & $24.5 \%$ & 0.001 \\
\hline IHD & $68 \%$ & $78.8 \%$ & $63.1 \%$ & 0.09 \\
\hline Heart failure & $38.4 \%$ & $45.5 \%$ & $35.5 \%$ & 0.31 \\
\hline Anemia & $75 \%$ & $91.2 \%$ & $68.8 \%$ & 0.005 \\
\hline Ulcer size $\left(>5 \mathrm{~cm}^{2}\right)$ & $68.6 \%$ & $73.5 \%$ & $65.6 \%$ & 0.39 \\
\hline Infection & $70.5 \%$ & $70.6 \%$ & $69.4 \%$ & 0.89 \\
\hline Osteomyelitis & $68.8 \%$ & $82.3 \%$ & $63.4 \%$ & 0.14 \\
\hline Heel Ulcer & $25.7 \%$ & $38.2 \%$ & $19.3 \%$ & 0.03 \\
\hline
\end{tabular}

HbA1c, glycated hemoglobin, type A1C; IHD, ischemic heart disease; PTA, percutaneous transluminal angioplasty; rePTA, repeated PTA.

cases, there was a restenosis of vessels treated at the first approach; in 4/74 (5.8\%) cases, there was a restenosis and a progression of $\mathrm{PAD}$ too (stenosis or occlusion of new vessel not affected at the first PTA). Superficial femoral artery (SFA) was treated for a second time in 40/243 (16.4\%) of cases, popliteal artery in 18/98 (18.3\%), tibial-peroneal trunk in $8 / 93(8.6 \%)$, anterior tibial artery in $23 / 182$ $(12.6 \%)$, peroneal artery in $8 / 98(7.8 \%)$, and posterior tibial artery in $20 / 148(13.5 \%)$.

Table 2 reports the arterial pattern disease of the whole population and separately of rePTA group and no rePTA group. At baseline, rePTA had more vessels affected, a higher involvement of peroneal, pedal, and plantar arteries than no rePTA group. Furthermore, rePTA group had lower increase of $\mathrm{TcPO}_{2}$ after endovascular intervention than no rePTA group.

\section{Outcomes}

rePTA patients had a lower rate of healing (28.5\% vs. $71.9 \%) p=0.0001$, higher rate of ulcer recurrence $(20 \%$ vs. $10.3 \%) p=0.03$, major amputation $(24.3 \%$ vs. $4.3 \%) p=0.0005$, and death $(33.3 \%$ vs. $7.9 \%)$ $p=0.002$ in comparison with no rePTA group (Fig. 1).

Time to amputation for rePTA and no rePTA group was, respectively, $2 \pm 0.7$ and $4.6 \pm 1.7$ months $(p=0.03)$.

Time to ulcer recurrence for rePTA and no rePTA group was, respectively, $6.7 \pm 2$ and $10.9 \pm 1.7$ months $(p=0.13)$.

At multivariate analysis of independent variables found at the univariate analysis, impaired glycemic control (glycated hemoglobin, type A1C [HbA1c] $)(2.2$ [1.9-2.7] $p=0.02)$ and dialysis (1.5 [1.4-3.6] $p=0.006$ ) were independent predictors of clinical recurrence of CLI.

Table 2. Arterial pattern disease of the whole population, rePTA group, and no rePTA group

\begin{tabular}{|c|c|c|c|c|}
\hline & General $(n=304)$ & rePTA $(n=74)$ & No rePTA $(n=230)$ & $p$ \\
\hline Vessels affected & $4.4 \pm 1.5$ & $5.1 \pm 1.6$ & $4.2 \pm 1.4$ & 0.001 \\
\hline Vessels treated at first PTA & $2.6 \pm 1.2$ & $2.4 \pm 1$ & $2.6 \pm 1.2$ & 0.5 \\
\hline Iliac & $12 / 304(3.9 \%)$ & $8 / 74(10.8 \%)$ & $4 / 230(1.7 \%)$ & 0.09 \\
\hline CFA & $2 / 304(0.6 \%)$ & $0 / 74(0 \%)$ & $2 / 230(0.8 \%)$ & 0.43 \\
\hline Profunda & $10 / 304(3.2 \%)$ & $0 / 74(0 \%)$ & $5 / 230(2.1 \%)$ & 0.09 \\
\hline SFA & 243/304 (79.9)\% & $60 / 74(81.1) \%$ & $183 / 230(79.5 \%)$ & 0.91 \\
\hline Popliteal & $106 / 304(34.8 \%)$ & $25 / 74(33.8 \%)$ & $81 / 230(35.2 \%)$ & 0.76 \\
\hline TTP & $101 / 304(33.2 \%)$ & $32 / 74(43.2 \%)$ & $69 / 304(22.7 \%)$ & 0.09 \\
\hline ATA & $262 / 304(86.2 \%)$ & $67 / 74(90.5 \%)$ & $195 / 230(84.8 \%)$ & 0.4 \\
\hline Peroneal artery & $170 / 304(55.9 \%)$ & $53 / 74(71.6 \%)$ & $117 / 230(50.8 \%)$ & 0.02 \\
\hline PTA & $248 / 304(81.5 \%)$ & $65 / 74(87.8 \%)$ & $183 / 230(79.5 \%)$ & 0.29 \\
\hline Pedal artery & $100 / 304(32.9 \%)$ & $34 / 74(45.9 \%)$ & $66 / 230(28.7 \%)$ & 0.03 \\
\hline Plantar Arteries & $107 / 304(35.2 \%)$ & $37 / 74(50 \%)$ & $70 / 230(30.4 \%)$ & 0.04 \\
\hline Subintimal approach & $52 / 304(17.1 \%)$ & $14 / 74(18.9 \%)$ & $38 / 230(16.7 \%)$ & 0.7 \\
\hline Stent & $57 / 304(18.7 \%)$ & $13 / 74(17.5 \%)$ & $44 / 230(19.1 \%)$ & 0.8 \\
\hline $\mathrm{TcPO}_{2} 1(\mathrm{mmHg})$ & $42.7 \pm 13.5$ & $32.5 \pm 11.3$ & $45.4 \pm 12.8$ & 0.0001 \\
\hline
\end{tabular}

ATA, anterior tibial artery; CFA, common femoral artery; SFA, superficial femoral artery; $\mathrm{TcPO}_{2}$, transcutaneous oxygen pressure; TTP, tibioperoneal trunk. 


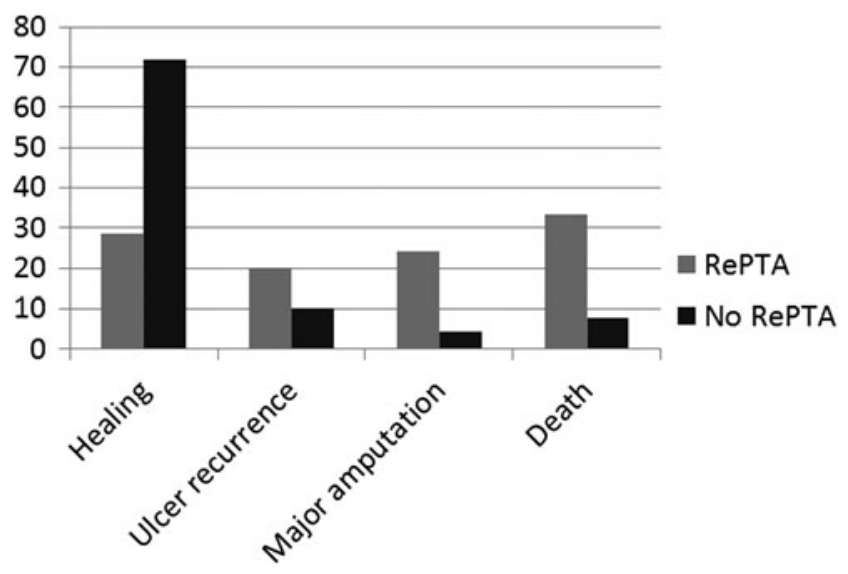

Figure 1. Outcomes in rePTA and no rePTA group. PTA, percutaneous transluminal angioplasty; rePTA, repeated PTA.

\section{DISCUSSION}

To our knowledge, there are few studies assessing the risk of clinical restenosis and the factors related to CLI relapse in diabetic patients with CLI with foot tissue loss. Faglia et al. described the extensive use of peripheral angioplasty and the risk of clinical recurrence of ischemia after PTA in diabetic patients with ischemic foot ulcer, even though specific risk factors related to restenosis were not described. ${ }^{8,13}$

The authors reported a low risk of restenosis in diabetic patients with ischemic foot ulcers treated by PTA: $7.3 \%$ after 4 months in a first study ${ }^{8}$ and $6.4 \%$ per year in a second one. ${ }^{13}$ In a similar group of patients treated by PTA, our group reported $9.6 \%$ of clinical ischemia recurrence in $\sim 20$ months of follow-up. ${ }^{7}$

Our data confirm that PTA is characterized by a certain risk of restenosis. We found that $\sim 24 \%$ of patients had rePTA because of the recurrence of CLI. In all cases, the reintervention was due to the restenosis of treated vessels. Probably the morphological rate of restenosis is higher than clinical CLI relapse. However, from our clinical point of view, when ulcer heals and pain disappears, the possible restenosis of treated vessels is not relevant.

We found a higher rate of clinical CLI relapse than data reported by Faglia (6.4 and $7.3 \%)$ and Uccioli (9.6\%); however, our cohort of subjects was characterized by several comorbidities, mainly dialysis. In fact, in our study, the rate of dialyzed patients is higher than in similar studies $(31.4 \% \mathrm{vs}$. $3.7 \%, 6.0 \%$ and $12.8 \%))^{7,8,13}$ In addition, dialysis resulted in an independent risk factor for clinical recurrence of CLI in the above mentioned patients. In our opinion, this element could justify the high rate of restenosis found in our population.
SFA and popliteal artery showed a higher rate of restenosis than infrapopliteal vessels, and the mean time to rePTA was $\sim 3.5$ months; therefore, the first months after revascularization should be considered the critical period for restenosis. rePTA patients showed a high risk of nonhealing, major amputation, and death, as this group was made up of subjects with more comorbidities that could influence the worst outcomes than no rePTA patients. However, already in other studies, a high risk of major amputation in patients with restenosis after revascularization has been reported. ${ }^{25}$ Furthermore, rePTA patients showed a high risk of ulcer recurrence than no rePTA patients.

We found that dialysis and high glycemic levels significantly increased the risk of rePTA. Dialysis is a well-recognized risk factor for PAD, and dialyzed patients have high risk of nonhealing, major amputation, and death. ${ }^{26-29}$ In our recent study, we already reported that dialyzed diabetic patients had a higher rate of rePTA when compared with the nondialyzed. ${ }^{30}$ Therefore, we can confirm that dialysis is a strong and specific risk factor of CLI relapse in diabetic patients with DFUs.

In relationship to glycemic levels, the association between glycemic control and outcomes has been extensively studied mainly for coronary artery procedure (balloon angioplasty). ${ }^{31-35}$ Both preprocedural $\mathrm{HbA} 1 \mathrm{c}$ and fasting blood glucose have been evaluated with mixed results. ${ }^{32,34}$ In fact, some studies highlighted the role of hyperglycemia as risk factor for restenosis after percutaneous coronary intervention, while the role of HbA1c and insulin resistance is less clear. ${ }^{36}$ The neointimal proliferation, after both balloon angioplasty and stenting, seems to contribute to the high rate of restenosis in diabetic patients. ${ }^{37}$ To our knowledge, only one study described the risk of restenosis in relationship to glycemic levels after lower limbs angioplasty. Singh et al. reported that high blood glucose levels ( $>144 \mathrm{mg} / \mathrm{dL}$ ) during infrapopliteal balloon angioplasty was associated with a significant reduction of primary patency and increased risk of major amputation in patients with CLI and tissue loss. ${ }^{38}$ Our data show that impaired glycemic control expressed by elevated values of $\mathrm{HbA} 1 \mathrm{c}$ is an independent risk factor for clinical recurrence of CLI in diabetic patients with DFUs treated by PTA. Although the data are still poor and the biological determinants of restenosis among patients with diabetes are not completely known, we retain that an adequate glycemic control during and after the angioplasty procedure could reduce the risk of neointimal proliferation, endothelial inflammation, and restenosis. Nevertheless, 
it is already reported that $\mathrm{HbA} 1 \mathrm{c}$ is a predictive factor for major amputation. ${ }^{7}$

Peripheral angioplasty is extensively used in our reality, and we have already reported in other studies the effectiveness of PTA in terms of limb salvage for ischemic DFUs. ${ }^{7,30}$ Furthermore, this procedure can be proposed with a low risk for fragile patients with several comorbidities. However, a significant risk of restenosis and clinical recurrence of CLI should be considered, mainly in dialyzed patients. The first months after the revascularization seem to be at high risk for restenosis and a great effort should be addressed to achieve wound healing in this first period. Furthermore, close postprocedural surveillance to optimize the durability of endovascular procedures by combining appropriate pharmacotherapy and periodic assessment of vessel patency is required. Nowadays, industrial efforts are directed to create new technical tools (low-profile balloons, drug-eluting balloons, medicated and nonmedicated stents) to ensure the long-term patency of the treated vessels. ${ }^{39-43}$ Finally, close clinical surveillance should be addressed to cardiometabolic risk factors and medical therapy after revascularization. Hypertension and dyslipidemia need to be treated, and dual antiplatelet therapy at least for 1 month after PTA is mandatory. Medical management with dual antiplatelet therapy and statin is recommended after vascular procedure, not only to reduce the risk of CLI recurrence but also to improve survival. ${ }^{44}$

\section{Innovation}

It is well known about the high rate of restenosis after PTA in diabetic patients with CLI and ischemic DFUs. This syudy is the first one that reports the correlation between clinical recurrence of CLI after endovascular revascularization and outcomes; furthermore, the clinical factors that independently influence the recurrence of CLI have been identified. Close monitoring of renal parameters and glycemic levels may reduce the rate of restenosis after PTA.

\section{ACKNOWLEDGMENTS AND FUNDING SOURCES}

No competing financial interests exist. The study follows the principles outlined in the Declaration of Helsinki.

\section{AUTHOR DISCLOSURE AND GHOSTWRITING}

No competing financial interests exist. The content of this article was expressly written by the authors listed. No ghostwriters were used to write this article.

\section{ABOUT THE AUTHORS}

Marco Meloni, MD, and Valentina Izzo, MD, are both $\mathrm{PhD}$ students and are currently involved in the medical and surgical treatment of diabetic foot along with Laura Giurato, MD, PhD, and Luigi Uccioli, MD. Costantino Del Giudice, MD, and Valerio Da Ros, MD, PhD, are currently involved in the treatment of vascular disease by endovascular approach. Roberto Gandini, MD, is the Chief of Interventional Radiology Department at University of Tor Vergata in Rome. Valerio Cervelli, MD, is the Chief of Plastic Surgery Department at University of Tor Vergata in Rome.

\section{REFERENCES}

1. Jude B, Eleftheriadou I, Tentolouris N. Peripheral arterial disease in diabetes. Diabet Med 2010;27: 4-14

2. Faglia E, Caravaggi C, Marchetti $R$, et al. Screening for peripheral arterial disease by means of the ankle-brachial index in newly diagnosed type 2 diabetic patients. Diabet Med 2005;22:1310-1314.

3. Prompers L, Schaper N, Apelqvist J, et al. Prediction of outcome in individuals with diabetic foot ulcers: focus on the differences between individuals with and without peripheral arterial disease. The EURODIALE Study. Diabetologia 2008;51:747-755.

4. Apelqvist J, Elgzyri T, Larsson J, Londahl M, Nyberg $P$, Thorne J. Factors related to outcome of neuroischemic/ischemic foot ulcer in diabetic patients. J Vasc Surg 2011;53:1582-1588.

5. McNeely MJ, Boyko EJ, Ahroni JH, et al. The independent contributions of diabetic neuropa- thy and vasculopathy in foot ulceration. How great are the risks? Diabetes Care 1995;18:216219.

6. Ferraresi R, Centola M, Ferlini M, et al. Long-term outcomes after angioplasty of isolated, belowthe-knee arteries in diabetic patients with critical limb ischaemia. Eur J Vasc Endovasc Surg 2009; $37: 336-342$.

7. Uccioli L, Gandini R, Giurato L, et al. Long-term outcomes of diabetic patients with critical limb 
ischemia followed in a tertiary referral diabetic foot clinic. Diabetes Care 2010;33:977-982.

8. Faglia E, Mantero M, Caminiti M, et al. Extensive use of peripheral angioplasty, particularly infrapopliteal, in the treatment of ischaemic diabetic foot ulcers: clinical results of a multicentric study of 221 consecutive diabetic subjects. J Intern Med 2002;252:225-232.

9. Jämsén T, Manninen $H$, Tulla $H$, Matsi P. The final outcome of primary infrainguinal percutaneous transluminal angioplasty in 100 consecutive patients with chronic critical limb ischemia. J Vasc Interv Radiol 2002;13:455-463.

10. Jacqueminet $S$, Hartemann-Heurtier A, Izzillo $R$, et al. Percutaneous transluminal angioplasty in severe diabetic foot ischemia: outcomes and prognostic factors. Diabetes Metab 2005;31:370375.

11. Bargellini I, Petruzzi P, Scatena A, et al. Primary infrainguinal subintimal angioplasty in diabetic patients. Cardiovasc Intervent Radiol 2008;31: 713-722.

12. Graziani L, Piaggesi A. Indications and clinical outcomes for below knee endovascular therapy: review article. Catheter Cardiovasc Interv 2010; 75:433-443.

13. Faglia E, Clerici G, Clerissi J, et al. Long term prognosis of diabetic patients with critical limb ischemia: a population based cohort study. Diabetes Care 2009;32:822-827.

14. Alexandrescu V, Hubermont G, Philips Y, et al Combined primary subintimal and endoluminal angioplasty for ischaemic inferior-limb ulcers in diabetic patients: 5 -year practice in a multidisciplinary "diabetic-foot" service. Eur J Vasc Endovasc Surg 2009;37:448-456.

15. Weis-Müller BT, Römmler V, Lippelt I, et al. Critical chronic peripheral arterial disease: does outcome justify crural or pedal bypass surgery in patients with advanced age or with comorbidities? Ann Vasc Surg 2011;25:783-795

16. Werneck CC, Lindsay TF. Tibial angioplasty for limb salvage in high-risk patients and cost analysis. Ann Vasc Surg 2009:23:554-559.

17. Pomposelli FB, Kansal N, Hamdan AD, et al. A decade of experience with dorsalis pedis artery by-pass: analysis of outcome in more than 1000 cases. J Vasc Surg 2003;37:307-315.

18. Pomposelli FB, Marcaccio EJ, Gibbons GW, et al. Dorsalis pedis arterial bypass: durable limb salvage for foot ischemia in patients with diabetes mellitus. J Vasc Surg 1995;21:375-384.

19. Capek P, McLean GK, Berkowitz HD. Femoropopliteal angioplasty. Factors influencing longterm success. Circulation 1991;83:170-180.

20. Romiti M, Albers M, Brochado-Neto FC, Durazzo $A E$, Pereira CA, De Luccia N. Meta-analysis of infrapopliteal angioplasty for chronic critical limb ischemia. J Vasc Surg 2008;47:975-981.

21. Faglia E, Clerici G, Clerissi J, et al. Angioplasty for diabetic patients with failing bypass graft or re- sidual critical ischemia after bypass graft. Eur $\mathrm{J}$ Vasc Endovasc Surg 2008:36:331-338.

22. Dick F, Diehm N, Galimanis A, Husmann M, Schmidli J, Baumgartner I. Surgical or endovascular revascularization in patients with critical limb ischemia: influence of diabetes mellitus on clinical outcome. J Vasc Surg 2007;45: 751-761.

23. Gandini R, Chiappa R, Di Primio M, et al. Recanalization of the native artery in patients with bypass failure. Cardiovasc Intervent Radiol 2009; 32:1146-1153.

24. Aiello A, Anichini R, Brocco E, et al. Treatment of peripheral arterial disease in diabetes: a consensus of the Italian Societies of Diabetes (SID, AMD), Radiology (SIRM) and Vascular Endovascular Surgery (SICVE). Nutr Metab Cardiovasc Dis 2014;24:355-369.

25. Sagib NU, Domenick N, Cho JS, et al. Predictors and outcomes of restenosis following tibial artery endovascular interventions for critical limb ischemia. J Vasc Surg 2013;57:692-699.

26. Ramdev P, Rayan SS, Sheahan M, et al. A decade experience with infrainguinal revascularization in a dialysis-dependent patient population. J Vasc Surg 2002;36:969-974.

27. Aulivola B, Gargiulo M, Bessoni M, Rumolo A, Stella A. Infrapopliteal angioplasty for limb salvage in the setting of renal failure: do results justify its use? Ann Vasc Surg 2005;19:762-768.

28. Rabellino M, Aragón-Sánchez J, González G, et al. Is endovascular revascularisation worthwhile in diabetic patients with critical limb ischemia who also have end-stage renal disease? Diabetes Res Clin Pract 2010;90:e79-e81.

29. Sigala F, Georgopoulos S, Langer S, et al. Outcomes of infrainguinal revascularization for critical limb ischemia in diabetics with end stage renal disease. Vasa 2006;35:15-20.

30. Meloni M, Giurato L, Izzo V, et al. Long term outcomes of diabetic haemodialysis patients with critical limb ischemia and foot ulcer. Diabetes Res Clin Pract 2016;116:117-122.

31. Ueda H, Mitsusada N, Harimoto K, Miyawaki M, Yasuga Y, Hiraoka H. Glycosylated hemoglobin is a predictor of major adverse cardiac events after drug-eluting stent implantation in patients with diabetes mellitus. Cardiology 2010;116:51-57.

32. Corpus RA, George PB, House JA, et al. Optimal glycemic control is associated with a lower rate of target vessel revascularization in treated type II diabetic patients undergoing elective percutaneous coronary intervention. J Am Coll Cardiol 2004; 43:8-14.

33. O'Connor DJ, Gargiulo NJ, Jang J. Hemoglobin A1c as a measure of disease severity and outcome in limb threatening ischemia. J Surg Res 2012;174:29-32.

34. Lemesle G, Bonello L, De Labriolle A, et al. Prognostic value of hemoglobin $\mathrm{A} 1 \mathrm{C}$ levels in patients with diabetes mellitus undergoing per- cutaneous coronary intervention with stent implantation. Am J Cardiol 2009:104:41-45.

35. Marfella R, Sasso FC, Siniscalchi M, et al. Periprocedural tight glycemic control during early percutaneous coronary intervention is associated with a lower rate of in-stent restenosis in patients with acute ST-elevation myocardial infarction. J Clin Endocrinol Metab 2012;97:2862-2871.

36. Lindsay J, Sharma AK, Canos D, et al. Preprocedure hyperglycemia is more strongly associated with restenosis in diabetic patients after percutaneous coronary intervention than is hemoglobin A1C. Cardiovasc Revasc Med 2007;8: 15-20.

37. Park SH, Marso SP, Zhou Z, Foroudi F, Topol EJ, Lincoff AM. Neointimal hyperplasia after arterial injury is increased in a rat model of non-insulindependent diabetes mellitus. Circulation 2001; 104:815-819

38. Singh S, Armstrong EJ, Sherif W, et al. Association of elevated fasting glucose with lower patency and increased major adverse limb events among patients with diabetes undergoing infrapopliteal balloon angioplasty. Vasc Med 2014;19:307-314.

39. White CJ, Gray WA. Endovascular therapies for peripheral arterial disease: an evidence-based review. Circulation 2007:116:2203-2215.

40. Schwarzwalder U, Zeller T. Below-the-knee revascularization. Advanced techniques. J Cardiovasc Surg 2009:50:627-634

41. Gandini R, Volpi T, Pampana E, et al. Applicability and clinical results of percutaneous transluminal angioplasty with a novel, long, conically shaped balloon dedicated for below-the knee interventions. J Cardiovasc Surg 2009;50:365-371.

42. Ferraresi R, Centola M, Biondi-Zoccai G. Advances in below-the-knee drug-eluting balloons $\mathrm{J}$ Cardiovasc Surg 2012;53:205-213.

43. Gandini R, Del Giudice C, Merolla S, Morosetti D, Pampana E, Simonetti G. Treatment of chronic SFA in-stent occlusion with combined laser atherectomy and drug-eluting balloon angioplasty in patients with critical limb ischemia: a singlecenter, prospective, randomized study. J Endovasc Ther 2013;20:805-814.

44. De Martino RR, Eldrup-Jorgensen J, Nolan BW, et al. Perioperative management with antiplatelet and statin medication is associated with reduced mortality following vascular surgery. J Vasc Surg 2014:59:1615-1621.

\section{Abbreviations and Acronyms}

$$
\begin{aligned}
\mathrm{CLI} & =\text { critical limb ischemia } \\
\mathrm{DFUS} & =\text { diabetic foot ulcers } \\
\mathrm{HbA} 1 \mathrm{c} & =\text { glycated hemoglobin, type } \mathrm{A} 1 \mathrm{C} \\
\mathrm{PAD} & =\text { peripheral arterial disease } \\
\mathrm{PTA} & =\text { percutaneous transluminal } \\
& \quad \text { angioplasty } \\
\text { rePTA } & =\text { repeated PTA } \\
\mathrm{SFA} & =\text { superficial femoral artery } \\
\mathrm{TCPO}_{2} & =\text { transcutaneous oxygen pressure }
\end{aligned}
$$

